\title{
EFEITO DO AQUECIMENTO SOBRE A FLEXIBILIDADE \\ E O DESEMPENHO FUNCIONAL: ENSAIO CLÍNICO RANDOMIZADO
}

\author{
EFFECT OFWARM-UP ON FLEXIBILITY AND FUNCTIONAL PERFORMANCE:RANDOMIZED CLINICAL TRIAL
}

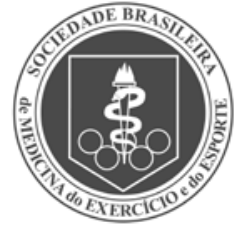

Artigo Original

Original Article Artículo Original

\section{EFECTO DEL CALENTAMIENTO SOBRE LA FLEXIBILIDAD Y EL RENDIMIENTO FUNCIONAL: ENSAYO CLÍNICO ALEATORIZADO}

Franklin Gadelha Cunha'

(Fisioterapeuta)

André Revoredo Assad

(Fisioterapeuta)

Talita Juliana de Carvalho Campos Pereira' (Fisioterapeuta)

Pedro Ivo de Souza Pinheiro²

(Fisioterapeuta)

Germanna de Medeiros Barbosa² (Fisioterapeuta)

Caio Alano de Almeida Lins² (Fisioterapeuta)

Bento João $\mathrm{Abreu}^{3}$

(Fisioterapeuta e Professor Associado)

Wouber Hérickson de Brito Vieira ${ }^{4}$

(Fisioterapeuta e Professor Adjunto)

1. Universidade Federal do Rio Grande do Norte, Natal, RN, Brasil. 2. Universidade Federal do Rio Grande do Norte, Programa de pósgraduação em Fisioterapia, Natal, RN, Brasil.

3. Universidade Federal do Rio Grande do Norte, Centro de Biociências, Departamento de Morfologia, Natal, RN, Brasil. 4. Universidade Federal do Rio Grande do Norte, Centro de Ciências da Saúde, Departamento de Fisioterapia, Natal, RN, Brasil.

\section{Correspondência:}

Wouber Hérickson de Brito Vieira. Universidade Federal do Rio Grande do Norte, Campus Universitário Av. Senador Salgado Filho, 3000, Lagoa Nova, Natal, RN,

Brasil. 59072-970.

hericksonfisio@yahoo.com.br

\section{RESUMO}

Introdução: A atividade física deve ser parte fundamental de programas de promoção da saúde. No entanto, sua realização pode expor o indivíduo a riscos de lesão, o que torna necessária a adoção de medidas preventivas como o aquecimento, com o intuito de minimizar os riscos e/ou contribuir para o melhor desempenho funcional. Objetivo: O propósito deste estudo foi analisar o efeito agudo de diferentes tempos de aquecimento sobre flexibilidade, equilíbrio e desempenho funcional em indivíduos fisicamente ativos. Método: Trinta e dois homens saudáveis que realizavam exercício regularmente pelo menos três vezes por semana foram aleatoriamente incluídos em um de quatro grupos ( $n=8)$ : G0 (sem aquecimento), G5 (aquecimento por cinco min.), G10 (aquecimento por dez min.) e G15 (aquecimento por quinze min.). Os indivíduos foram avaliados antes e depois da intervenção nas seguintes variáveis: flexibilidade do reto femoral (RF) e dos isquiotibiais (IT), equilíbrio corporal com olhos abertos e fechados e desempenho funcional por meio dos testes de salto triplo horizontal (STH) e shuttle run (SR). O aquecimento foi realizado numa esteira ergométrica entre $70 \%$ e $80 \%$ da frequência cardíaca máxima estimada para idade. Resultado: Não houve diferenças significativas na flexibilidade e no equilíbrio nas comparações intra e intergrupos $(p>0,05)$. Contudo, houve melhora significativa do desempenho funcional somente no G10 na comparação intragrupo para as variáveis STH (de 5,88 \pm 0,55 para 6,23 \pm 0,66; $p=0,0051$ ) e SR (de 4,72 $\pm 0,13$ para 4,61 $\pm 0,13 ; p=0,0194$ ). Conclusão: $O$ aquecimento durante 10 minutos parece melhorar o desempenho funcional em indivíduos ativos, podendo também ser uma alternativa viável para a prevenção de lesão.

Descritores: atividade motora; saúde; temperatura corporal; esforço físico.

\section{ABSTRACT}

Introduction: Physical activity should be a fundamental part of health promotion programs. However, its performance can expose the individual to risk of injury, which makes it necessary to adopt preventive measures such as warm-up $(W U)$ in order to minimize risks and/or contribute to better functional performance. Objective: The purpose of this study was to analyze the acute effect of different warm-up times on flexibility, balance, and functional performance in physically active individuals. Method: Thirty two healthy men, who exercise regularly at least three times a week, were randomly assigned to one of four groups ( $n=8$ ): G0 (without WU), G5 (WU for $5 \mathrm{~min}$ ), G10 (WU for $10 \mathrm{~min}$ ), and G15 (WU for $15 \mathrm{~min}$ ). The subjects were assessed before and after the intervention on the following variables: flexibility of the rectus femoris (RF) and hamstrings (HM) muscles, body balance with open and close eyes and functional performance through triple horizontal jump (THJ) and shuttle run (SR) tests. The WU was carried out on a treadmill between $70 \%$ and $80 \%$ estimated maximum heart rate for age. Results: There were no significant differences in flexibility and balance in intra and intergroup comparisons ( $p>0.05$ ). However, there was a significant improvement in functional performance only in G10 in the intragroup comparison for THJ variables $(5.88 \pm 0.55$ to $6.23 \pm 0.66 ; p=0.0051)$ and $S R$ variables ( $4.72 \pm 0.13$ to $4.61 \pm 0.13 ; p=0.0194)$ variables. Conclusion: Warm-up for 10 minutes seems to improve functional performance in active individuals, and may be a viable alternative for injury preventions.

Keywords: motor activity; health; body temperature; physical exertion.

\section{RESUMEN}

Introducción: La actividad física debe ser parte fundamental de los programas de promoción de la salud. Sin embargo, su realización puede exponer al individuo a riesgos de lesión, lo que hace necesaria la adopción de medidas preventivas como el calentamiento con el fin de minimizar los riesgos y/o contribuir para el mejor desempeño funcional. Objetivo: El propósito de este estudio fue analizar el efecto agudo de diferentes tiempos de calentamiento sobre flexibilidad, equilibrio y desempeño funcional en individuos físicamente activos. Método: Treinta y dos hombres sanos que hacen ejercicio regularmente por lo menos tres veces por semana fueron incluidos aleatoriamente en uno de cuatro grupos ( $n=8)$ : G0 (sin calentamiento), G5 (calentamiento por cinco minutos), G10 (calentamiento por diez minutos) y G15 (calentamiento por quince minutos). Los individuos fueron evaluados antes y después de la intervención 
en las siguientes variables: flexibilidad del recto femoral (RF) y de los isquiotibiales (IT), equilibrio corporal con ojos abiertos y cerrados y desempeño funcional por medio de las pruebas de salto triple horizontal (STH) y shuttle run (SR). El calentamiento se realizó en una cinta rodante entre el 70\% y el 80\% de la frecuencia cardiaca máxima estimada para la edad. Resultado: No hubo diferencias significativas en la flexibilidad y el equilibrio en las comparaciones intra e intergrupos ( $p>0,05)$. Sin embargo, hubo una mejora significativa en el desempeño funcional sólo en el G10 en la comparación intragrupo para las variables STH (de 5,88 $\pm 0,55$ a 6,23 $\pm 0,66, p=0,0051$ ) y $S R$ (de 4,72 $\pm 0,13$ a 4,61 $\pm 0,13, p=0,0194$ ). Conclusión: El calentamiento durante 10 minutos parece mejorar el desempeño funcional en individuos activos, pudiendo también ser una alternativa viable para la prevención de lesión.

Descriptores: actividad motora; salud; temperatura corporal; esfuerzo físico.

\section{INTRODUÇÃO}

É consenso que saúde e qualidade de vida podem ser preservadas e aprimoradas pela prática regular de atividade física, já que esta combate o sedentarismo ${ }^{1}$. Nesse sentido, sugere-se que a atividade física deve ser parte fundamental de programas mundiais de promoção da saúde ${ }^{2}$. No entanto, a realização de exercícios pode expor o praticante a riscos de lesão ${ }^{3}$, sendo necessária a adoção de medidas preventivas com o intuito de minimizar os riscos e/ou contribuir para o melhor desempenho funcional.

Dentre essas medidas, o aquecimento é popularmente utilizado na prática de atividades físicas e desportivas como exercícios preparatórios para treinos ou competições ${ }^{4}$, apresentando uma variedade de tipos, formas e tempos de realização. Segundo Weineck ${ }^{5}$, o principal objetivo do aquecimento corporal ativo é obter aumento da temperatura corporal e muscular, assim como preparar os sistemas cardiovascular e pulmonar para o desempenho motor. Assim, o indivíduo poderia obter adequação do estado físico e psíquico, tal como a preparação cinética e coordenativa para a prevenção de lesões ${ }^{1}$.

Dessa forma, o aquecimento geral deve ser ativo, não muito intenso, envolvendo principalmente os músculos que serão utilizados durante a execução do exercício ${ }^{5}$. Assim, seriam promovidas alterações fisiológicas diversas tais como a diminuição da viscosidade muscular, aumento da velocidade de transmissão nervosa, contração muscular e elevação da temperatura corporal ${ }^{6}$. Além disso, tem sido destacado os efeitos psicológicos que se manifestam principalmente com a sensação de "estar preparado" e a capacidade do aquecimento simular os movimentos específicos de determinada atividade física ${ }^{3,5,7}$.

De acordo com as diretrizes internacionais para a prescrição e aplicação de testes de esforço, o aquecimento deve ser realizado entre cinco e 10 minutos numa intensidade entre $60-80 \%$ do consumo máximo de oxigênio $\left(\mathrm{VO}_{2 \text { máx }}\right)$, seguido por um período de recuperação menor ou igual a 10 minutos $^{7}$ ou em uma intensidade abaixo do exercício físico que será realizado. Entretanto, a melhor estratégia de aquecimento ainda não está bem estabelecida dada a presença de algumas variáveis como duração, intensidade, e tipo de atividade realizado, as quais podem ser determinantes na sua eficácia ${ }^{8}$.

Dessa forma, apesar do aquecimento fazer parte de programas de atividade física de diferentes populações e possuir informações relevantes na literatura quanto aos seus benefícios, as variações de intensidade e/ou de tempo na prática esportiva dificulta a comparação entre resultados de diferentes estudos ${ }^{9}$. As informações disponíveis são controversas quanto aos desfechos das práticas do aquecimento, as quais podem proporcionar efeitos agudos positivos e/ou negativos em avaliações motoras subsequentes ${ }^{10}$.

Portanto, o objetivo do estudo foi investigar o efeito agudo de diferentes tempos de aquecimento sobre a flexibilidade, equilíbrio e desempenho funcional em indivíduos ativos.

\section{MATERIAIS E MÉTODOS}

Trinta e dois sujeitos do sexo masculino, idade média de $23 \pm 5$ anos, estatura 1,75 \pm 5 m e índice de massa corporal 21,75 $\pm 3,25 \mathrm{~kg} / \mathrm{m}^{2}$ participaram deste estudo. Todos indivíduos realizavam atividade física regular e recreacional envolvendo os membros inferiores (MMII) por pelo menos três vezes por semana, há aproximadamente três meses. Nenhum dos sujeitos apresentava patologia musculoesquelética nos MMII há pelo menos seis meses anteriormente ao estudo, assim como outras alterações cardíacas, distúrbios neurológicos e/ou vestibulares. Foram excluídos os que não completaram algum procedimento experimental no decorrer do estudo $(n=6)$. Todos assinaram um termo de consentimento livre e esclarecido, de acordo com a Resolução 466/12 do Conselho Nacional de Saúde e a Declaração de Helsinki, antes da admissão no experimento. O estudo foi aprovado pelo comitê de ética em pesquisa local (Protocolo 163.539) e desenvolvido no Departamento de Fisioterapia da Universidade Federal do Rio Grande do Norte (UFRN).

\section{Procedimentos}

Inicialmente, foi realizado o preenchimento da ficha de avaliação cinético-funcional dos MMII. Em seguida, os indivíduos foram designados, por meio do site wWw.randomization.com, para um dos quatro grupos experimentais, identificados por diferentes cores: grupo $0(\mathrm{G} 0 ; \mathrm{n}=8)$ foi o controle, apenas submetido às avaliações inicial e final realizadas antes e após o período de intervenção; grupo 5 (G5; $n=8), 10(\mathrm{G} 10 ; \mathrm{n}=8)$ e 15 $(\mathrm{G} 15 ; \mathrm{n}=8)$ que, além das avaliações, foram submetidos a um minuto de caminhada e 5, 10 e 15 minutos de aquecimento na esteira ergométrica (Esteira Eletrônica E-710 - Movement ${ }^{\circledR}$ - Brasil), respectivamente.

Os procedimentos do estudo foram realizados por três avaliadores: I (responsável pelo processo de aleatorização da amostra e intervenção), II (responsável pelas avaliações) e III (responsável pela análise estatística). Todos os procedimentos do estudo foram realizados no turno vespertino, entre 15 e 18 horas, sob as mesmas condições laboratoriais $\left(25^{\circ} \mathrm{C}\right)$.

\section{Avaliação da flexibilidade}

A flexibilidade dos músculos ísquiotibiais (IT) foi avaliada conforme citado por Shuback et al. ${ }^{11}$. Foi utilizado um goniômetro universal (Carci ${ }^{\circledR}$ - Brasil), uma mesa de exame e um dispositivo de madeira especialmente construído para posicionamento e fixação do sujeito. O indivíduo era posicionado em decúbito dorsal com os membros superiores (MMSS) sobre o tórax. O quadril e joelho do membro inferior dominante era flexionado a $90^{\circ}$ (Figura 1A). O voluntário era estabilizado por meio de cintas na pelve e membro inferior contralateral e orientado a movimentar a perna de modo ativo em direção à extensão do joelho, com o goniômetro posicionado conforme citado por Chagas et al. ${ }^{12}$. O déficit de extensão do joelho era calculado como a diferença entre o ângulo de extensão do joelho mensurado e o ângulo de 180 , considerado o ângulo de extensão máxima normal desta articulação. 
A flexibilidade do reto femoral (RF) foi realizada por meio do teste de Thomas modificado ${ }^{13}$ (Figura 1B). O indivíduo era posicionado em decúbito dorsal com os MMIl pendentes sobre a mesa de exame e solicitado a realizar a flexão do quadril e joelho do lado não-dominante. Registrava-se os ângulos inicial e final de extensão passiva do joelho contralateral (dominante) por meio de um goniômetro.

Ambos os testes eram realizados três vezes consecutivas, no membro dominante, obtendo-se a média ao final da avaliação.

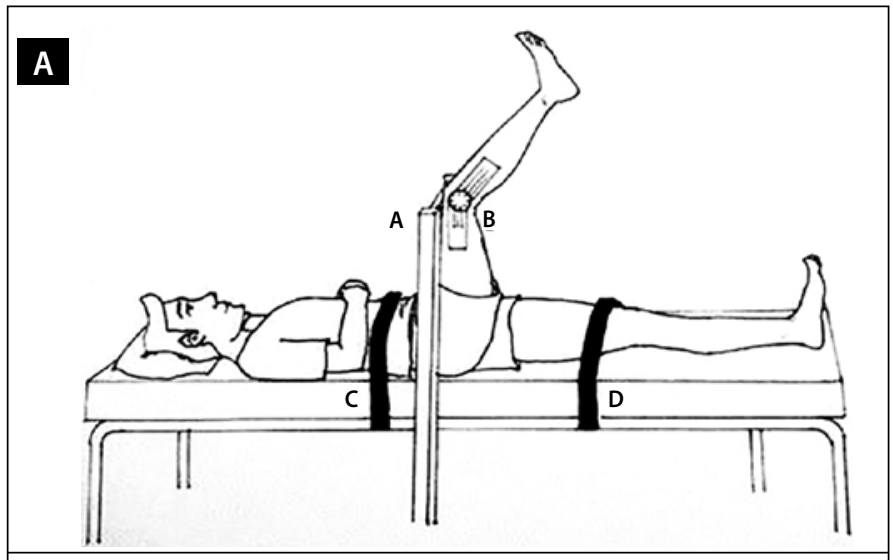

B

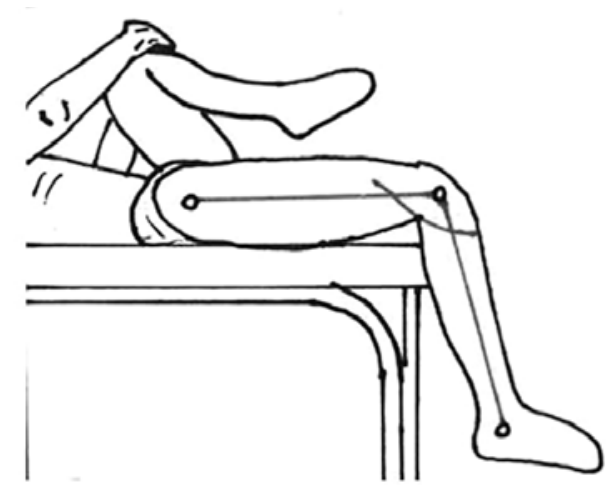

Figura 1. A) Avaliação da flexibilidade dos isquiotibiais (IT). A - Dispositivo para limitar a flexão do quadril a 90\%; B - Mensuração do ângulo poplíteo utilizando o goniômetro universal; C e D- Faixa de velcro para fixação da pelve e coxa contralateral, respectivamente. B) Avaliação da flexibilidade do Reto Femoral (RF) - Teste de Thomas modificado.

\section{Avaliação do equilíbrio}

Para avaliação do equilíbrio foi utilizado o aparelho Balance Master System (NeuroCom ${ }^{\oplus}$ Balance Manager Systems \& Products, USA). Foi realizado o teste de apoio unipodal no membro dominante (unilateral stance), avaliando-se o equilíbrio estático em uma superfície estável por 10 segundos, com os olhos abertos e fechados. Os indivíduos realizavam o teste com os pés descalços, joelho do membro dominante estendido enquanto o joelho contralateral era flexionado a $90^{\circ}$, e MMSS encontravam-se juntos ao longo do corpo. Inicialmente realizava-se a familiarização com o procedimento e equipamento. Após cinco minutos, eram realizadas três repetições de cada teste. $O$ intervalo de repouso era de um minuto entre as tentativas, considerando-se o valor médio ${ }^{14,15}$.

\section{Avaliação do desempenho funcional}

Para a avaliação do desempenho funcional foram utilizados consecutivamente os testes de salto triplo horizontal (STH) e o shuttle run (SR).

No primeiro teste, específico para se avaliar a distância máxima alcançada ${ }^{16}$, os sujeitos foram posicionados com o hálux tocando a linha de demarcação de início do salto, em apoio unipodal do membro dominante, e no momento que julgavam propício, realizavam três saltos consecutivos, com os MMSS livres para auxiliar na impulsão. Mensurava-se a distância do salto conforme citado por Holm et al. ${ }^{17}$.

Para o segundo teste, foi utilizado uma célula fotoelétrica modelo Speed Test Fit' ${ }^{18}$ (CEFISE, SP/Brasil), posicionada no ponto de partida (0 metro), de modo que o feixe de luz incidisse na crista ilíaca do avaliado, cronometrando o percurso com precisão em milésimos de segundo.

O teste foi executado em uma superfície plana, onde os sujeitos colocaram-se em afastamento ânteroposterior dos membros, o mais próximo da linha, na qual estava localizada um dos cones e a célula fotoelétrica (0 metro). Com o comando verbal, percorreram uma distância total de 20 metros (10 metros de ida e 10 metros de volta), no menor tempo possível. Ambos os testes funcionais foram realizados três vezes, intervalo de 30 segundos de recuperação entre as tentativas, obtendo-se o maior valor.

\section{Protocolo de aquecimento}

Primeiramente, os indivíduos realizavam caminhada na esteira, com velocidade de $4 \mathrm{Km} / \mathrm{h}$ durante um minuto, como forma de evitar a transição abrupta repouso-aquecimento. Em seguida, o aquecimento era realizado na intensidade da esteira em $\mathrm{Km} / \mathrm{h}$ correspondente a 70-80\% da frequência cardíaca máxima ( $\mathrm{FC}_{\text {máx }}$ ) estimada para a idade do voluntário de acordo com a fórmula de Karvonen (220 - idade). A frequência cardíaca (FC) foi monitorada por um monitor cardíaco digital (Polar FT1 - EUA). A fim de se avaliar a percepção do esforço ao longo do aquecimento, foi utilizada também a escala de Borg (6 a 20).

Para manter o"cegamento" do estudo, todos os voluntários permaneceram 16 minutos na sala de aquecimento com o avaliador responsável, já que correspondia ao tempo do maior aquecimento entre os grupos.

\section{Análise estatística}

A análise dos dados foi realizada por meio do Statistical Package for the Social Science (SPSS ${ }^{\circledR}$ ) versão 20.0. Inicialmente, foi aplicado o teste de normalidade de Shapiro Wilk para a verificar a distribuição dos dados e o teste de Levene, para a análise da homogeneidade das variâncias. Em seguida, foi realizado a ANOVA (two-way) para verificação de diferenças intra e inter-grupo. Quando necessário, foi utilizado o teste post-hoc de Tukey para a identificação das diferenças. Os dados foram expressos por meio da média e desvio-padrão com nível de significância $p \leq 0,05$.

\section{RESULTADOS}

A caracterização da amostra está descrita na Tabela 1.

Pode-se observar que não houve diferença significativa nos valores iniciais para as variáveis antropométricas (Anova $p>0,05$ ). Por outro lado, pode-se observar que tanto a percepção de esforço quanto a FC aumentaram de forma significativa com a progressão do tempo de aquecimento $(p<0,05)$.

Em relação à flexibilidade dos músculos IT e RF, bem como a variável equilíbrio (Tabela 2), pode-se observar que não houve diferenças significativas nas comparações intra e inter-grupos $(P>0,05)$.

Em relação ao desempenho funcional (Tabela 2), observa-se na comparação intragrupo que houve um incremento somente no G10 para ambas as variáveis - STH $(p=0,0051)$ e $S H(p<0,0194)$. Entretanto, não houve diferenças significativas na comparação intergrupo $(p>0,05)$.

Tabela 1. Caracterização da amostra quanto aos dados antropométricos e biológicos.

\begin{tabular}{c|c|c|c|c|c}
\hline Variáveis & G0 & G5 & G10 & G15 & \\
\hline & Média $( \pm)$ & Média $( \pm)$ & Média $( \pm)$ & Média $( \pm)$ & Anova \\
\hline Idade (anos) & $22,63 \pm 2,72$ & $20,75 \pm 1,98$ & $22,13 \pm 1,45$ & $23,28 \pm 1,84$ & $\mathrm{p}=0,097$ \\
\hline Peso $(\mathrm{kg})$ & $69,50 \pm 6,88$ & $67,25 \pm 7,05$ & $67,88 \pm 5,35$ & $71,44 \pm 5,53$ & $\mathrm{P}=0,549$ \\
\hline Altura (M) & $1,74 \pm 0,05$ & $1,75 \pm 0,05$ & $1,75 \pm 0,05$ & $1,74 \pm 0,05$ & $\mathrm{P}=0,950$ \\
\hline $\mathrm{IMC}\left(\mathrm{Kg} / \mathrm{m}^{2}\right)$ & $22,99 \pm 1,47$ & $21,91 \pm 1,93$ & $22,22 \pm 2,19$ & $23,54 \pm 1,09$ & $\mathrm{P}=0,246$ \\
\hline Borg & $6 \pm 0$ & $9 \pm 2,14$ & $8 \pm 2,10$ & $10 \pm 2,34$ & $\mathrm{P}=0,0006^{*}$ \\
\hline $\mathrm{FC}(\mathrm{bpm})$ & $91 \pm 5,13$ & $147 \pm 9,03$ & $147 \pm 5$ & $159 \pm 3,59$ & $\mathrm{P}=0,0001^{*}$ \\
\hline
\end{tabular}


Tabela 2. Média e desvio-padrão das variáveis flexibilidade, equilíbrio e desempenho funcional antes e após o período de aquecimento nos diferentes grupos.

\begin{tabular}{|c|c|c|c|c|c|c|c|c|c|c|c|c|c|}
\hline Variáveis & \multicolumn{3}{|c|}{ G0 } & \multicolumn{3}{|c|}{ G5 } & \multicolumn{3}{|c|}{ G10 } & \multicolumn{3}{|c|}{ G15 } & \multirow[b]{2}{*}{ Anova } \\
\hline & Inicial & Final & teste $t$ & Inicial & Fi Final & teste $t$ & Inicial & Final & teste $t$ & Inicial & Final & teste $t$ & \\
\hline $\begin{array}{c}\text { Isquiotibiais } \\
\text { (graus) }\end{array}$ & $151,9 \pm 11,42$ & $152,1 \pm 9,26$ & 0,8606 & $156,3 \pm 5,52$ & $159,0 \pm 3,82$ & 0,076 & $153,5 \pm 6,82$ & $155,6 \pm 5,04$ & 0,3300 & $154,3 \pm 8,95$ & $152,9 \pm 9,84$ & 0,4425 & 0,6362 \\
\hline $\begin{array}{l}\text { Reto Femoral } \\
\text { (graus) }\end{array}$ & $1,8 \pm 2,66$ & $2,1 \pm 2,59$ & 0,5490 & $2,5 \pm 1,69$ & $1,9 \pm 2,53$ & 0,405 & $3,4 \pm 1,77$ & $2,3 \pm 1,75$ & 0,2931 & $3,0 \pm 3,817$ & $2,0 \pm 3,423$ & 0,1546 & 0,1423 \\
\hline Equil. $\mathrm{AO}(\% / \mathrm{s})$ & $0,7 \pm 0,18$ & $0,7 \pm 0,16$ & 0,5490 & $0,7 \pm 0,21$ & $0,8 \pm 0,16$ & 0,1705 & $0,6 \pm 0,10$ & $0,7 \pm 0,12$ & 0,0675 & $0,7 \pm 0,14$ & $0,8 \pm 0,16$ & 0,3051 & 0,223 \\
\hline Equil. OF $(\% / s)$ & $1,3 \pm 0,33$ & $1,4 \pm 0,33$ & 0,6821 & $1,6 \pm 0,49$ & $1,5 \pm 0,43$ & 0,5538 & $1,5 \pm 0,33$ & $1,4 \pm 0,35$ & 0,3424 & $1,6 \pm 0,32$ & $1,5 \pm 0,23$ & 0,4060 & 0,296 \\
\hline $\begin{array}{c}\text { Salto triplo } \\
\text { horizontal } \\
\text { (metros) }\end{array}$ & $5,85 \pm 0,40$ & $5,96 \pm 0,41$ & 0,0668 & $6,36 \pm 0,28$ & $6,49 \pm 0,24$ & 0,272 & $5,88 \pm 0,55$ & $6,23 \pm 0,66$ & $0,0051^{* *}$ & $6,11, \pm 0,51$ & $6,27 \pm 0,38$ & 0,2342 & 0,266 \\
\hline $\begin{array}{l}\text { Shuttle run } \\
\text { (segundos) }\end{array}$ & $4,78 \pm 0,23$ & $4,73 \pm 0,20$ & 0,2526 & $4,62 \pm 0,19$ & $4,54 \pm 0,13$ & 0,074 & $4,72, \pm 0,13$ & $4,61, \pm 0,13$ & $0,0194^{*}$ & $4,76, \pm 0,22$ & $4,71 \pm 0,21$ & 0,0879 & 0,397 \\
\hline
\end{tabular}

\section{DISCUSSÃO}

Uma das respostas clínicas em decorrência do aquecimento é uma maior extensibilidade da unidade musculotendínea e diminuição da rigidez, o que proporcionaria um aumento da amplitude de movimento (ADM) e menores riscos de lesões musculares ${ }^{19}$. No entanto, o presente estudo demonstrou que não houve alteração significativa na flexibilidade após o aquecimento.

Uma das justificativas para este resultado envolve o tipo de aquecimento utilizado e, consequentemente, o grau de elevação da temperatura. Weineck et al. ${ }^{5}$ citaram que a temperatura entre 38,8 e 41,6C é tida como apropriada para se atingir a plasticidade das fibras musculares. No entanto, apesar de não ser verificada a temperatura corporal no presente estudo, o tempo de aquecimento estabelecido nos três grupos pode não ter sido suficiente para alcançar estes valores com alterações significativas em ambos os grupos musculares. Além disso, a elevação de temperatura decorrente do aquecimento não ocorre de forma imediata no músculo, o que pode dificultar sua contribuição no aumento da $\mathrm{ADM}^{8}$.

Uma das contribuições mais importantes do aquecimento é minimizar índices de lesões músculoesqueléticas durante as atividades físicas e esportivas, e melhorar o senso de posição corporal e os parâmetros de equilíbrio ${ }^{20}$. No presente estudo, não foram observadas alterações significativas no equilíbrio nas condições realizadas. Num estudo semelhante realizado por Subasi et al. ${ }^{21}$, investigou-se os efeitos agudos de cinco e 10 minutos de aquecimento na propriocepção do joelho e no equilíbrio, com os olhos abertos e fechados. Ao contrário do presente estudo, os autores encontraram efeitos positivos sobre as variáveis supracitadas. Além disso, 0 aquecimento de 10 minutos apresentou valores significativos na melhora da propriocepção se comparados aos de 5 minutos.

Bouet et al. ${ }^{22}$ apresentaram resultados semelhantes após 10 minutos de aquecimento submáximo em bicicleta ergométrica. De acordo com os autores, os exercícios de aquecimento são moderados e mantém a frouxidão em níveis normais, podendo prevenir lesões por melhorar a sensibilidade de mecanorreceptores.

Da mesma forma, a velocidade de condução do impulso nervoso aumenta em função do aquecimento ${ }^{23}$, podendo contribuir para uma maior velocidade de reação, coordenação dos movimentos ${ }^{24}$ e consequentemente maior força, sendo o aumento de $2^{\circ} \mathrm{C}$ correspondente ao aumento de $20 \%$ da velocidade de contração ${ }^{5}$. Um dos motivos para isso ocorrer é a diminuição do tempo de abertura-fechamento dos canais $\mathrm{Na}^{+} / \mathrm{K}^{+}$, proporcionando um menor tempo para as trocas iônicas nas células e tendo como consequência a diminuição do potencial de ação. Isto pode influir diretamente no tempo de latência muscular ${ }^{23}$, possibilitando uma ativação mais rápida e que resultaria numa maior contração dos sarcômeros.

Possivelmente, uma das razões para o aquecimento não ter influenciado os parâmetros de equilíbrio no presente estudo refere-se ao fato de não ter sido suficiente para promover alterações significativas na temperatura dos componentes neurais, o que resultaria em uma maior velocidade de condução nervosa e, consequentemente, num menor tempo de reação.

Essa hipótese pode ser corroborada pelo fato dos indivíduos do estudo serem ativos e saudáveis, e assim, não apresentarem déficits neurais no que se refere à acuidade proprioceptiva. Outro aspecto relevante refere-se ao tipo de avaliação realizada, a qual foi estática, diferentemente de outros estudos que avaliaram também a forma dinâmica. Importante ressaltar que após a intervenção do aquecimento, o equilíbrio estático foi a última variável a ser reavaliada, sugerindo que os efeitos do aquecimento podem ter sido "perdidos" pelo maior tempo decorrido até a reavaliação.

O presente estudo constatou uma melhora significativa intragrupo apenas no G10. Segundo Weineck ${ }^{5}$, aumentos rápidos na temperatura muscular alcançam um limiar na curva tempo x temperatura por volta de 10 a 15 minutos após o início do exercício, enquanto o aumento da temperatura central ocorre de forma mais gradual, ao longo de um período de 30 minutos. Tais dados estão de acordo com os achados do presente estudo, onde os melhores resultados foram encontrados no G10.

Asmussen e Boje ${ }^{24}$ encontraram melhores resultados no sprint de ciclismo quando a temperatura muscular aumentou acentuadamente e a central ficou próxima a valores basais. Os autores concluíram que a temperatura muscular era o fator mais importante que influenciava no desempenho funcional. Embora não tenha sido mensurada diretamente no presente estudo, o aumento da temperatura muscular pode ser a responsável pela melhora do desempenho, provocando melhoria na fluidez e na eficácia do gesto esportivo ${ }^{6,25}$.

Em relação aos demais grupos, pode-se inferir que, no G0, a ausência de incremento significativo advém do fato dos indivíduos não aquecerem, e consequentemente não prepararem o corpo para o exercício ${ }^{5}$. Já no G5, possivelmente o tempo para a plena ativação das vias energéticas não foi suficiente, e não atingiu o "steady state" de $\mathrm{VO}_{2}{ }^{26}$. No G15, provavelmente os voluntários apresentaram leve cansaço e fadiga muscular, levando em consideração que esse trabalho não foi realizado em atletas.

\section{CONCLUSÃO}

O presente estudo observou que o aquecimento não influenciou a flexibilidade dos músculos IT e RF o equilíbrio estático. Por outro lado, o G10 apresentou melhora no desempenho funcional ao comparar os momentos pré e pós-aquecimento. Vale ressaltar que os resultados do presente estudo devem limitar-se a homens ativos, que praticam atividade física regularmente e de forma moderada. Assim, sugere-se que novos estudos avaliem os efeitos de diferentes aquecimentos, tanto em indivíduos com alterações neuromusculares ou em atletas profissionais, já que estão expostos a estímulos maiores.

Todos os autores declararam não haver qualquer potencial conflito de interesses referente a este artigo. 
CONTRIBUIÇÕES DOS AUTORES: Cada autor contribuiu individual e significativamente para o desenvolvimento do manuscrito. FGC (0000-0002-5279-5613)*, ARA (0000-0002-9246-1909)* , TJCCP (0000-0002-1971-7998)* e PISP (0000-0003-3110-2897)* participaram da elaboração e desenho do tema, coleta, análise e interpretação dos dados, pesquisa bibliográfica e discussão dos resultados. GMB (0000-0002-1094-334X)* e CAAL (0000-0001-6424-3114)* participaram da discussão dos resultados, redação, análise estatística e revisão crítica do conteúdo intelectual do manuscrito. BJA (0000-0001-8010-806X)* e WHBV (0000-0003-3588-5577)** contribuiram na análise, discussão e revisão final e conceito intelectual do manuscrito. *ORCID (Open Researcher and Contributor ID).

\section{REFERÊNCIAS}

1. Carvalho T, Nóbrega ACL, Lazzoli JK, Magni JRT, Rezende L, Drummond FA. Posição oficial da Sociedade Brasileira de Medicina do Esporte: atividade física e saúde. Rev Bras Med Esport. 1996;2(4):79-81.

2. American College of Sports Medicine; Chodzko-Zajko WJ, Proctor DN, Fiatarone Singh MA, Minson CT, Nigg CR, et al. American College of Sports Medicine position stand. Exercise and physical activity for older adults. Med Sci Sports Exerc. 2009;41(7):1510-30.

3. Clebis NK, Natali MJM. Lesões musculares provocadas por exercícios excêntricos. Rev Bras Ciên Mov. 2001;9(4):47-53.

4. Bishop D. Warm up I: potential mechanisms and the effects of passive warm up on exercise performance. Sports Med. 2003;33(6):439-54

5. Weineck J. Treinamento ideal. 9a. ed. São Paulo: Manole; 2003

6. Woods K, Bishop P, Jones E. Warm-up and stretching in the prevention of muscular injury. Sports Med. 2007;37(12):1089-99.

7. American College of Sports Medicine. Diretrizes do ACSM para os Testes de Esforço e sua Prescrição. $6^{\text {a. }}$ ed. Rio de Janeiro: Guanabara Koogan; 2003.

8. Di Alencar TAM, Matias KFS. Princípios Fisiológicos do Aquecimento e Alongamento Muscular na Atividade Esportiva. Rev Bras Med Esporte. 2010;16(3):230-34

9. Fradkin AJ, Zazryn TR, Smoliga JM. Effects of warming-up on physical performance: a systematic review with meta-analysis. J Strength Cond Res. 2010;24(1):140-8.

10. Ribeiro YS, Del Vechio FB. Metanálise dos efeitos agudos do alongamento na realização de corridas curtas de alta intensidade. Rev Bras Educ Fís. Esporte. 2011;25(4)567-81.

11. Shuback B, Hooper J, Salisbury L. A comparision of a self- -stretch incorporating proprioceptive neuromuscular facilitation components and a therapist-applied PNF-technique on hamstring flexibility. Physiother. 2004; 90(3):151-7.

12. Chagas HM, Bhering EL, Bergamini JC, Menzel HJ. Comparison of two different stretching intensities in the range of motion. Rev Bras Med Esporte. 2008;14(2):99-103.

13. de Lucena GL, dos Santos Gomes C, Guerra RO. Prevalence and associated factors of Osgood-Schlatter syndrome in a population-based sample of Brazilian adolescents. Am I Sports Med. 2011:39(2):415-20.
14. Chuang SH, Huang MH, Chen TW, Weng MC, Liu CW, Chen CH. Effect of knee sleeve on static and dynamic balance in patients with knee osteoarthritis. Kaohsiung J Med Sci. 2007;23(8):405-11.

15. Citaker S, Kaya D, Yuksel I, Yosmaoglu B, Nyland J, Atay OA, et al. Static balance in patients with patellofemoral pain syndrome. Sports Health. 2011;3(6):524-7.

16. Neeb TB, Aufdemkampe G, Wagener JH, Mastenbroek L. Assessing anterior cruciate ligament injuries: the association and differential value of questionnaires, clinical tests, and functional tests. J Orthop Sports Phys Ther. 1997;26(6):324-31.

17. Holm I, Fosdahl MA, Friis A, Risberg MA, Myklebust G, Steen H. Effect of neuromuscular training on proprioception, balance, muscle strength, and lower limb function in female team handball players. Clin J Sport Med. 2004;14(2):88-94.

18. American Alliance for Health, Physical Education, and Recreation. Youth fitnesse test manual. Washington, DC: AAFHPER; 1976

19. Rosa AC, Montandon I. Efeitos do aquecimento sobre a amplitude de movimento: uma revisão crítica. Rev Bras Ciên Mov. 2006;14(1):109-16

20. Thacker SB, Stroup DF, Branche CM, Gilchrist J, Goodman RA, Porter Kelling E. Prevention of knee injuries in sports. A systematic review of the literature. J Sports Med Phys Fitness. 2003:43(2):165-79.

21. Subasi SS, Gelecek N, Aksakoglu G. Effects of different warm-up periods on knee proprioception and balance in healthy young individuals. J Sport Rehabil. 2008;17(2):186-205.

22. Bouët V, Gahéry Y. Muscular exercise improves knee position sense in humans. Neurosci Lett. 2000;289(2):143-6.

23. Rutkove SB. Effects of temperature on neuromuscular electrophysiology. Muscle Nerve. 2001;24(7):867-82 24. Asmussen WD, Boje O. Body temperature and capacity for work. Acta Physiol Scand. 1945;10:1-22.

25. Robergs RA, Roberts SO. Princípios fundamentais de fisiologia do exercício para aptidão, desempenho e saúde. São Paulo: Phorte; 2002

26. Baldari C, Guidetti L. A simple method for individual anaerobic threshold as predictor of max lactate steady state. Med Sci Sports Exerc. 2000:32(10):1798-802. 